\title{
Comparison of Extreme Surface Elevation for Linear and Nonlinear Random Wave Theory for Offshore Structures
}

\author{
Nurul ‘Azizah Mukhlas ${ }^{1,}$, Noor Irza Mohd Zaki ${ }^{1}$, Mohd Khairi Abu Husain ${ }^{1}$ and \\ Gholamhossein Najafian ${ }^{2}$ \\ ${ }^{1}$ Universiti Teknologi Malaysia, 54100 Kuala Lumpur, Malaysia \\ ${ }^{2}$ School of Engineering, Universiti of Liverpool, Liverpool, United Kingdom
}

\begin{abstract}
For offshore structural design, the load due to wind-generated random waves is usually the most important source of loading. While these structures can be designed by exposing them to extreme regular waves (100-year design wave), it is much more satisfactory to use a probabilistic approach to account for the inherent randomness of the wave loading. This method allows the statistical properties of the loads and structural responses to be determined, which is essential for the risk-based assessment of these structures. It has been recognized that the simplest wave generation is by using linear random wave theory. However, there is some limitation on its application as some of the nonlinearities cannot be explained when higher order terms are excluded and lead to underestimating of 100-year wave height. In this paper, the contribution of nonlinearities based on the second order wave theory was considered and being tested at a variety of sea state condition from low, moderate to high. Hence, it was proven that the contribution of nonlinearities gives significant impact the prediction of 100 -year wave's design as it provides a higher prediction compared to linear wave theory.
\end{abstract}

\section{Introduction}

An offshore structure is subjected to several categories of loads; accidental, permanent, deformation, live, and environmental loads [1]. However, as according to the API standard design code (2014) for fixed offshore platforms, an extreme environmental condition is the main parameter in formulating the platform design load according to its service life. In the North Sea, the wind generated random wave loads are the major consideration in the design stage since wind load itself only contribute less than $5 \%$ of total environmental loading [2]. The reliability analysis of fixed offshore structure can be measured either through deterministic or probabilistic approach. While these structures can be designed by exposing them to extreme regular waves (100-year design wave), it is much more satisfactory to use a probabilistic approach to account for the inherent randomness of the wave loading.

\footnotetext{
*Corresponding author: nurulazizahmukhlas@,gmail.com
} 
In the probabilistic approach, the response analysis of structure can be categorized into two; a fatigue failure limit state (FLS) and a total collapse limit state (ULS). In FLS, the analysis was done under normal conditions with a calm sea state [3]. Exceeding the given limits requires the termination of platform operations. A longer analysis is required for the safety condition by applying the ULS based on the first excursion failure. Normally, a 100year return period is required, where data on extreme wave conditions is needed according to the 100-year wave height record [4]. The wave-induced loads on the particular structures can be determined using Morison's equation since it has been widely applied in the last decades [5]. The waves are very descriptive as seen in nature. It can be explained based on its relative amplitudes, as small amplitude waves and finite amplitude waves.

A linear wave theory also known as small-amplitude wave theory, allowed boundary condition to be linearized. It is the simplest and most frequently used wave theory. However, it is well known that there existed a nonlinearity of the wave loading due to the drag component which gives a significant effect on both the frequency spectrum and probability distribution of the extreme value responses [6,7]. Furthermore, structural dynamics effects, the presence of current and load intermittency in the splash zone also increase the complexity of the problem. This provided limitation on the application of linear wave theory since some of the nonlinearities cannot be explained when higher order terms are excluded. In such situations, finite amplitude wave or nonlinear wave theory is more applicable to be applied.

In this respect, the contribution of higher-order Stokes-types terms to the linear approximation make the solution of the equation more complex and difficult, it is however provided a better result in term of accuracy of prediction extreme value responses [8]. The addition of at least the second-order nonlinear term is sufficient to yield a more accurate prediction compared to the linear solution [9]. It was proved by Forristall [10] that do the comparison between the second-order simulation in predict the extreme wave crest and highest wave crest obtained using appropriate instruments based on measured data. Both results agree well with each other. The second-order principle was initiated by Hasselmann, Longuet-Higgins, and Stewart [11-13] by considering the effect of wave steepness, water depth, and directional spreading. It was then being simplified by Dean, and Sharma \& Dalzell $[14,15]$ in term of the coefficient of second-order correction term for its application from deep, intermediate to shallow water condition $[10,16,17]$. In shallow water, the nonlinearity should be expected once its experienced extreme sea condition.

To this end, Alberello et. al. [9] and Chen [17] have compared the behavior of wave statics (i.e skewness and kurtosis) between linear and nonlinear wave theory on the secondorder term. The incident of wave train produces non-zero skewness showing non-gaussian properties with kurtosis value more than 3. While, Adcock [18] agreed that the magnitude of second order contribution involved both summation and difference correction term, consist of sum and difference of frequency between two linear wave. As a result, they produce the sharpening of the crests and flattening of the troughs.

In this study, the behavior of wave generation according to linear and nonlinear wave theory is discussed. The result will focus on the evaluation of extreme surface elevation and it is sufficient enough to describe the extreme response since those two are linear correlated $[19,20]$. The following section is devoted to a brief description on the superposition of waves that detailed on the wave generation based on linear theory. Followed by the wave interaction as one of the factors of nonlinearities in the real sea state and the contribution of second-order correction term in formulating the nonlinear wave theory. Finally, the result of wave generation using both theories was performed and the corresponding 100-year wave was being compared. 


\section{Superposition of waves}

An ocean wave is originally viewed as a sinusoidal shape having a small amplitude and is often described as a Gaussian distribution. Linear wave theory [21] is the simplest and most frequent model being used. The sea surface elevation $\eta(x, y, t)$ is treated as a summation of contributions of a large number of elementary waves with various frequencies, $w_{m}$ and directions, $\theta_{n}\left(0 \leq \theta_{n} \leq 2 \pi\right)$, superimposed randomly with:

$$
\begin{gathered}
\eta(x, y, t)=\sum_{m} \sum_{n} a_{m n} \cos \phi_{m n} \\
\phi_{m n}=k_{m}\left[x \cos \left(\theta_{n}\right)+y \sin \left(\theta_{n}\right)\right]-w_{m} t+\varphi_{m n}
\end{gathered}
$$

The water surface elevation is measured from the mean water level (MWL), where $x$ and $y$ refer to the point coordinate along the horizontal plane and $t$ is the time. The wave amplitude, $a_{m n}$ is a random variable according to the frequency, $w_{m}$ and direction, $\theta_{n}$. The phase angle, $\varphi_{m n}$ is assumed to be a random variable that uniformly distributed in the interval $0,2 \pi$. The wave number, $k_{m}$ is related to a wave frequency, $w_{m}$ by linear dispersion relation:

$$
w_{m}^{2}=g k_{m} \tanh \left(k_{m} d\right)
$$

where $g$ is the gravitational acceleration and $d$ is the water depth from the seabed to the surface elevation.

According to Massel [22], the superimposed of $\eta_{1}, \eta_{2}, \eta_{3}, \ldots, \eta_{N}$ are statistically independent random variables with the same distribution of mean and variance. This reflects that all the nonlinear interactions between wave component can be neglected. The wave surface profile, $\eta_{l}(x, y, t)$ from $\mathrm{Eq}(1)$ change the coordinate, $x$ and $y$ from time to time. However, by assuming that the wave impact on a fixed structure $\left(x_{0}, y_{0}\right)$ the wave profile only represents the function of time, simplified into the following equation:

$$
\begin{aligned}
\eta_{l}\left(x_{0}, y_{0}, t\right) & =\eta(t) \\
& =\sum_{m} a_{m} \cos \left(w_{m} t+\varphi_{m n}\right)
\end{aligned}
$$

However, in the real condition, the wave tend to behave differently with a finite amplitude having a peaked crest and flatten trough compare to that predicted using linear theory. This happened due to its nonlinear effect. The wave phenomenon is one of the reason. It is based on the interaction between two or more wave, or with fixed or free object.

\subsection{Wave interaction and Interference}

In general, wave interaction occur when the wave interacts with matter in several ways. Results in the reflection, diffraction or refraction of waves. In the case of water wave, the interaction happened when its hits an obstacle, e.g offshore structure. In the formation of sea water profile, only reflection and diffraction of interaction waves are considered. Refraction will only happen when wave pass through two mediums with different density. While diffraction give significant impact when the structure consist of large component, e.g bigger diameter of leg platform with $\pi D / L>0.15$, otherwise it is negligible. Hence, common interaction that occurred are wave reflection, where the wave will bounce it back. Depend on what was hitting is how it bounced back. If it is a fixed object, it will be 
inverted when it comes back and this will be considered in the formation of second order wave profile. If it is a free object, the opposite happened. According to Newton's third law, the fixed must be exerting an equal downward force when the wave is bounced back.

This reflection cause interference between two waves and tend to produce a larger wave. It can either be constructive or destructive. The illustration of the wave phenomenon can be observed in Fig. 1. A simbucket simulation has been carried out by Andersen [23] to illustrates the interface of two waves moving back and forth (refer Fig. 2). The blue wave is moving to the right, once it hits a fixed object, it reflects back and moving to the left which represent the red wave. As they move, they are interfering each other (green wave) and can be either in phase or out of phase. This phenomenon initially caused a nonlinearity on a random wave and it becomes worst with the presence of current that leads to drag dominant.

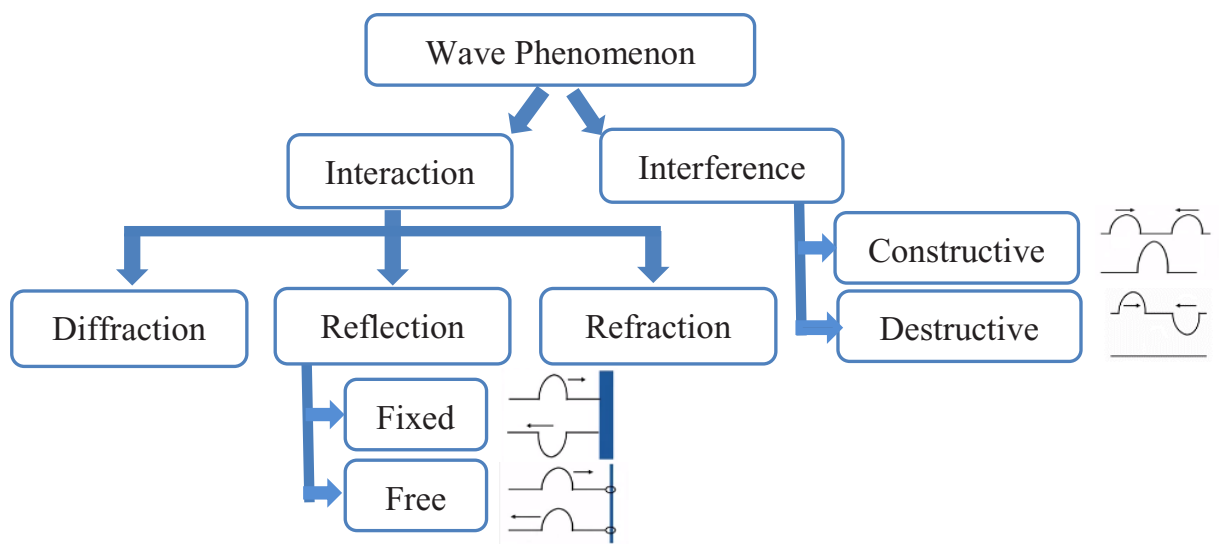

Fig. 1. Wave Phenomenon

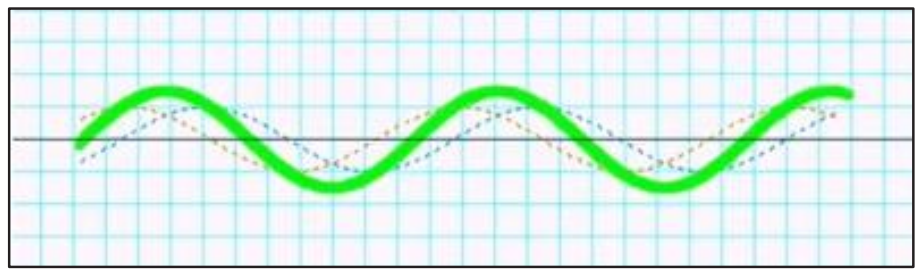

Fig. 2. Nonlinear Wave using Simbucket Simulation [23]

\section{Second order irregular waves}

The linear wave generation can be corrected by considering the second order correction term. It is an extension from Dean and Sharma [14], [24] that develop a model of secondorder nonlinearities that include all the important elements such as the effect of wave steepness, water depth, and directional spreading. The model has been applied by Forristall, Chen, Adcock and Toffoli et al. [10, 17, 18, 26]in formulating nonlinear wave component for an accurate prediction of wave loads.

In formulating the second order irregular waves, lets assumed that it happens with an interaction of two wave component having $\left(a_{i}, k_{i}, \omega_{i}\right)$ and $\left(a_{j}, k_{j}, \omega_{j}\right)$. The bichromatic 
wave spectrum experienced small spaced between the components, with $\Delta k=0.1 k$ [9]. Creating two terms of summation and difference frequencies :

$$
\begin{gathered}
\eta_{2+}=\sum_{i=1}^{N} \sum_{j=1}^{N} \alpha_{i} \alpha_{j} B_{P} \cos \left(\varphi_{i}+\varphi_{j}\right) \\
\eta_{2-}=\sum_{i=1}^{N} \sum_{j=1}^{N} \alpha_{i} \alpha_{j} B_{m} \cos \left(\varphi_{i}-\varphi_{j}\right)
\end{gathered}
$$

where $i$ and $j$ indicates first and second component. $N$ is the total harmonic waves. $B_{P}$ and $B_{m}$ are the interaction kernel of sum and difference term in the function of the interacting frequencies and water depth. Consist of two wave component with $\theta$ as an angle between the component, randomly between 0 to $\pi / 2$ :

$$
\text { summation term, } B_{P}=\alpha-\frac{\beta_{P} \gamma_{P}}{\delta_{P}}+\frac{\varepsilon_{P} \xi_{P}}{\delta_{P}}
$$

where,

$$
\begin{aligned}
\alpha & =\frac{\omega_{i}^{2}+\omega_{j}^{2}}{2 g} \\
\beta_{p} & =\frac{\omega_{i} \omega_{j}}{2 g}\left(1-\frac{\cos \theta}{\tanh \left(\left|k_{i}\right| d\right) \tanh \left(\left|k_{j}\right| d\right)}\right) \\
\gamma_{p} & =\left(\omega_{i}+\omega_{j}\right)^{2}+g\left|k_{i}+k_{j}\right| \tanh \left(\left|k_{i}+k_{j}\right| d\right) \\
\delta_{p} & =\left(\omega_{i}+\omega_{j}\right)^{2}-g\left|k_{i}+k_{j}\right| \tanh \left(\left|k_{i}+k_{j}\right| d\right) \\
\varepsilon_{p} & =\frac{\omega_{i}+\omega_{i}}{2 g} \\
\xi_{p} & =\frac{\omega_{i}^{3}}{\sinh ^{2}\left(\left|k_{i}\right| d\right)}+\frac{\omega_{j}^{3}}{\sinh ^{2}\left(\left|k_{j}\right| d\right)} \\
\text { and different term, } B_{m} & =\alpha+\frac{\beta_{m} \gamma_{m}}{\delta_{m}}+\frac{\varepsilon_{m} \xi_{m}}{\delta_{m}}
\end{aligned}
$$

where,

$$
\begin{aligned}
\beta_{m} & =\frac{\omega_{i} \omega_{j}}{2 g}\left(1+\frac{\cos \theta}{\tanh \left(\left|k_{i}\right| d\right) \tanh \left(\left|k_{j}\right| d\right)}\right) \\
\gamma_{m} & =\left(\omega_{i}-\omega_{j}\right)^{2}+g\left|k_{i}-k_{j}\right| \tanh \left(\left|k_{i}-k_{j}\right| d\right) \\
\delta_{m} & =\left(\omega_{i}-\omega_{j}\right)^{2}-g\left|k_{i}-k_{j}\right| \tanh \left(\left|k_{i}-k_{j}\right| d\right) \\
\varepsilon_{p} & =\frac{\omega_{i}-\omega_{j}}{2 g} \\
\xi_{p} & =\frac{\omega_{i}^{3}}{\sinh ^{2}\left(\left|k_{i}\right| d\right)}-\frac{\omega_{j}^{3}}{\sinh ^{2}\left(\left|k_{j}\right| d\right)}
\end{aligned}
$$


As a result, the surface elevation can be written as a second-order Stokes series, with the combination of linear wave and second-order correction term:

$$
\eta_{n l}(x, t)=\eta_{l}+\eta_{2+}+\eta_{2-}
$$

Figs. 3 and 4 are the sample of surface elevation represent both linear and nonlinear random wave theory. The result represents wave generation for 4.5 hours with $\pi / 2$ and 0 as an angle between the two components, $\theta .0$ angle indicates that both component having same direction. As proven by the previous researcher [26-27], the inclusion of nonlinear term result on the peaked crest and flattens trough and eventually effect on the design of 100year wave height which will be discussed in the next section.

Fig. 3 shows that both linear and nonlinear waves profile are almost correlated well. Meanwhile, for Fig.4, the nonlinear wave profile is unsteady and not smooth as linear wave showing that when two wave component move in the same direction $\left(\theta=0^{\circ}\right)$, the nonlinear wave profile does not correlated well with that using linear approach. However, it still followed the pattern of linear wave profile and its unsteady line indicates nonlinearity where the models considered the effects of wave steepness, water depth, and directional spreading with no approximation other than the truncation of a small amplitude expansion to the second order. Same output has been generated by Majewski et al. [28] in generating significant wave height as one of dthe atabase created for wave field characteristic at Arctic region.

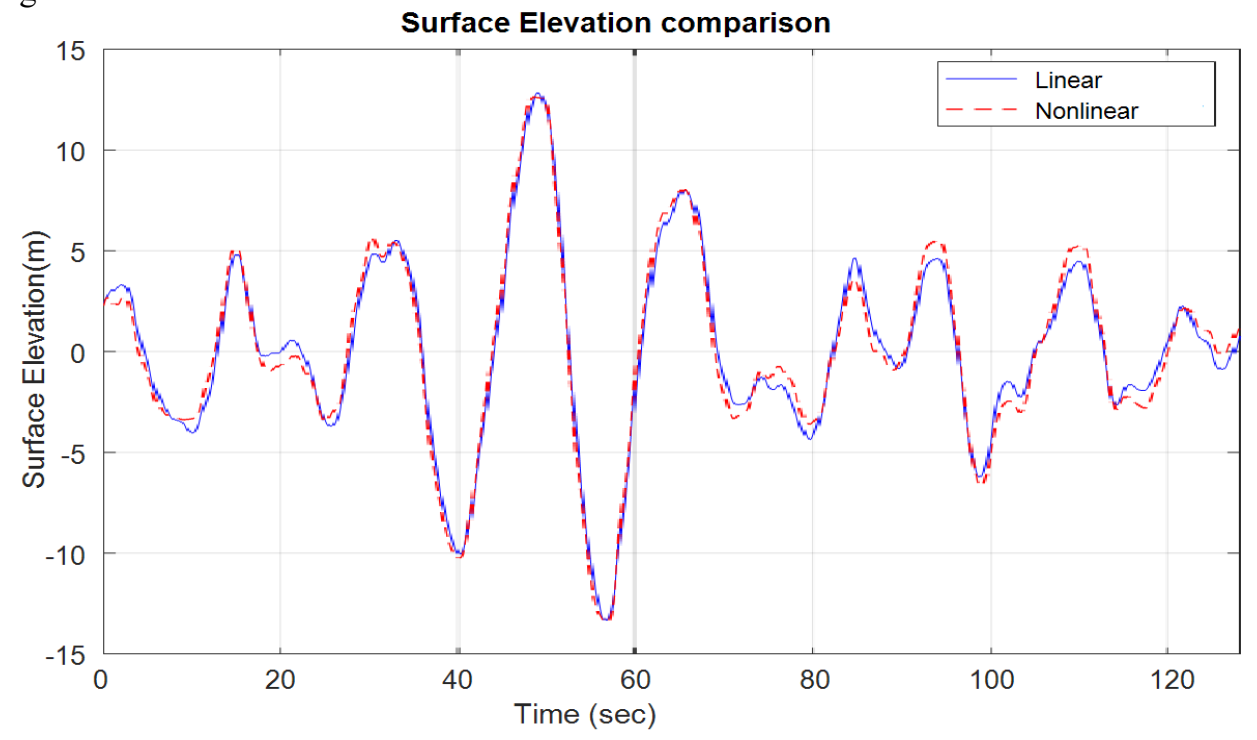

Fig. 3. Comparison of Linear and Nonlinear Surface Elevation for $H_{s}=15 \mathrm{~m}, T_{z}=13.75 \mathrm{sec}, \theta=\pi / 2$ and duration $=4.5$ hours. 


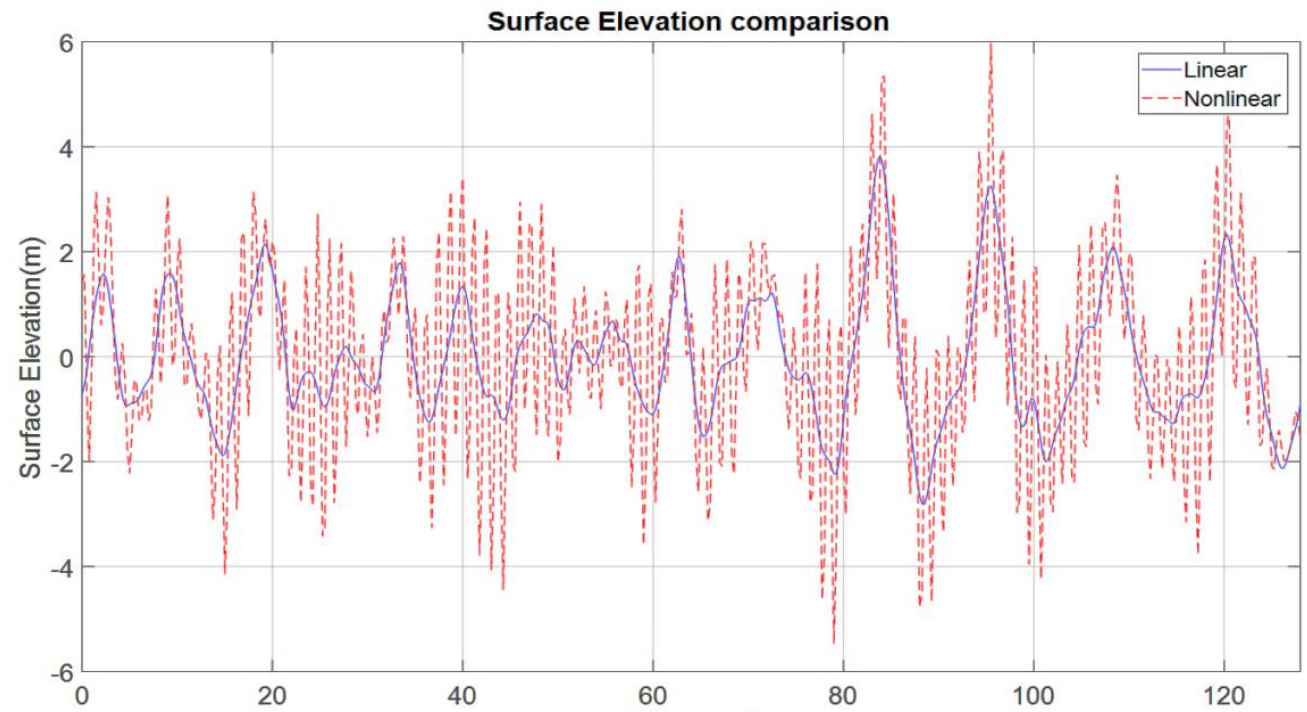

Fig. 4. Comparison of Linear and Nonlinear Suirfaedserevation for $H_{s}=5 \mathrm{~m}, T_{z}=13.75 \mathrm{sec}, \theta=0$ and duration $=4.5$ hours.

\section{Short-term probability distribution of extreme surface elevation}

The short-term probability distribution of extreme surface elevation is obtained using Monte Carlo time simulation operational framework. The procedure is illustrated in Fig. 5 and summarized as follows :

1. Identify the appropriate frequency wave spectrum (i.e. Pierson-Moskowitz spectrum) according to the site selection.

$$
G_{\eta \eta}(\omega)=\frac{H_{s}^{2}}{4 \pi T_{z}^{4} f^{5}} \exp -\left(\frac{1}{\pi T_{z}^{4} f^{4}}\right)
$$

where $G_{\eta \eta}(\omega)$ indicates surface elevation frequency spectrum, $f$ refer to the frequency of the wave, $H_{s}$ is the significant wave height and $T_{z}$ stands for mean zero upcrossing period.

2. Generate surface elevation of linear random wave theory using Eq. (4) by FFT for the purposed of simplification in obtaining realistic irregular sea waves.

3. Compute second-order correction term by determining the interaction kernel for both summation and difference terms. Refer Eqs. (5)-(8).

4. Generate surface elevation of nonlinear random wave theory with the combination of its linear random wave theory and the second order correction term as in Eq. (9).

5. Determine the extreme surface elevation for both linear and nonlinear random wave theory.

6. Repeat procedure 2 to 5 many times to generate a large sample of extreme surface elevation.

7. The recorded extreme values need to be arranged accordingly from the smallest to the largest numerical order.

8. Determine the corresponding probability distribution using plotting position equation for Rayleigh distribution, 


$$
\operatorname{Prob}=\left(r_{\max }<q_{n}\right)=P_{r_{\max }}\left(q_{n}\right) \approx \frac{n-0.44}{N+0.12}, \quad n=1,2,3, \ldots, N
$$

where $r_{\max }$ indicates the response extreme values, $q_{n}$ is the $n^{\text {th }}$ smallest simulated extreme value and $N$ shows the amount of all simulated extreme values.

9. Interpolate the 100-year wave height for a short-term probability distribution.

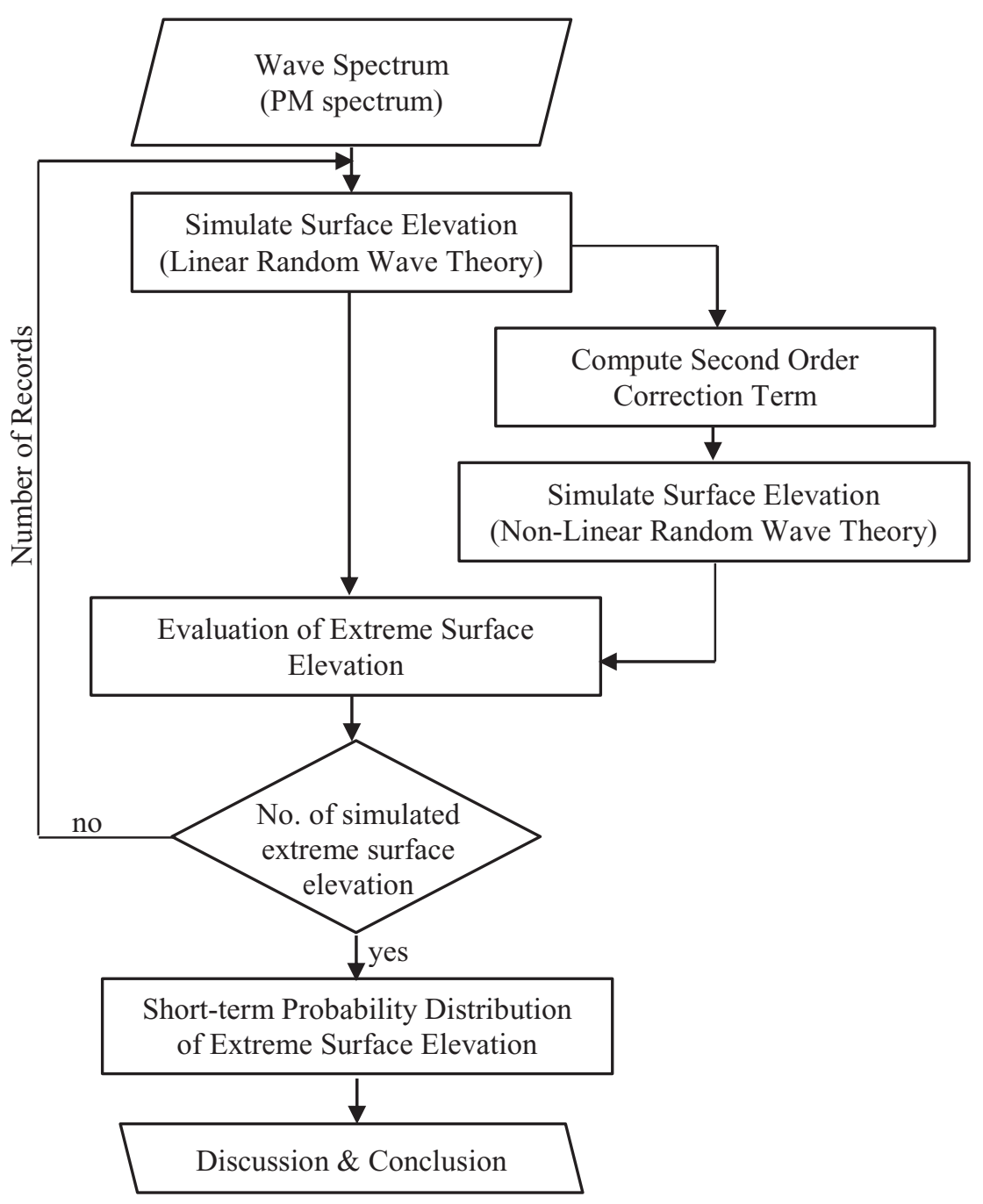

Fig. 5. Evaluation of short-term probability distribution of extreme surface elevation using Monte Carlo operational framework

As an example, Figs. 6 and 7 plotted the probability distribution of extreme surface elevation for both linear and nonlinear random wave theory to the Rayleigh scale at $H_{s}=$ $15 \mathrm{~m}$ and $H_{s}=15 \mathrm{~m}$, respectively. Monte Carlo simulation was being applied based on 10,000 realizations, each with random amplitude and phase angle. Initially, the wave simulation was evaluate according to linear random wave theory and later the contribution of second order term was taken into consideration for a prediction of 100 -year wave height. 


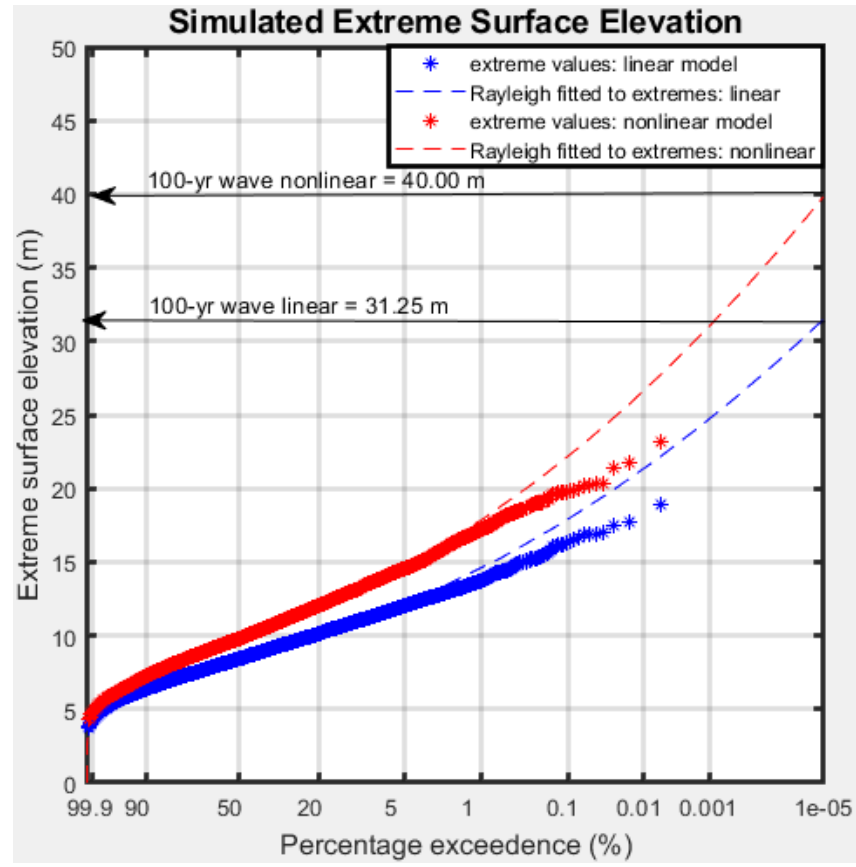

Fig. 6. Probability distribution of extreme surface elevation using linear and nonlinear wave theory for $H_{S}=15 \mathrm{~m}, T_{Z}=13.75 \mathrm{sec}$ and duration $=128 \mathrm{sec}$ with 10,000 simulation.

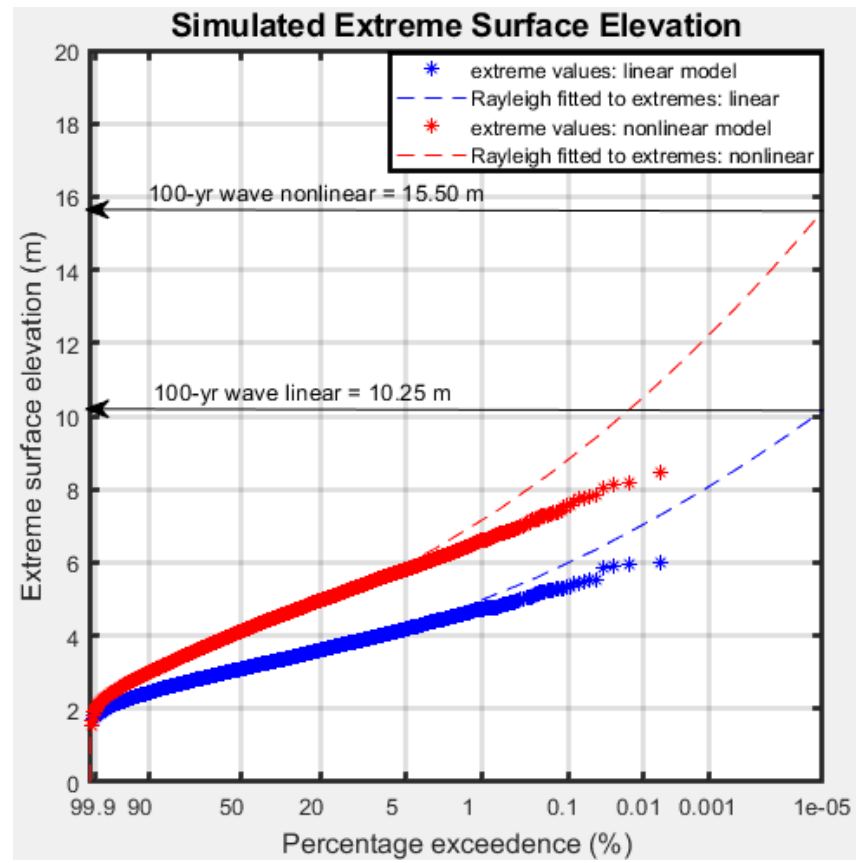

Fig. 7. Probability distribution of extreme surface elevation using linear and nonlinear wave theory for $H_{S}=5 \mathrm{~m}, T_{Z}=7.94 \mathrm{sec}$ and duration $=128 \mathrm{sec}$ with 10,000 simulation. 


\section{Results \& discussion}

Table 1 has summarized the prediction of 100-year wave height based on linear and nonlinear random wave theory and its corresponding ratio. By positioning result from linear random wave theory as a benchmark, the corresponding ratio is calculated. With a value of unity indicates that the prediction using both theories agree well with each other. While more than 1.0 shows that the linear random wave theory underestimates the prediction, in line with the finding by Zheng [6] claiming that linear random approach leads to response extreme values underestimate. Three different sea state from low, moderate to high $\left(H_{S}=5\right.$, $10 \& 15 \mathrm{~m}$ ) has been tested in this study to see the impact of nonlinearities in the evaluation of extreme surface elevation at variety sea state condition. Hence, according to the results, the prediction of 100-year wave height using linear random wave theory is lower than that using nonlinear random wave theory at all the sea state with the highest gap at low sea state giving around $50 \%$ under predicted.

Table 1. Comparison of 100-year wave height from linear and nonlinear models

\begin{tabular}{|c|c|c|c|}
\hline \multirow{2}{*}{$\begin{array}{l}H_{s} \\
(\mathbf{m})\end{array}$} & \multicolumn{2}{|c|}{ 100-year wave height (m) } & \multirow{2}{*}{ Ratio $=\frac{100-y r \text { wave }}{100-y r \text { wave }}$} \\
\hline & LRWT & NLRWT & \\
\hline 5 & 10.250 & 15.500 & 1.512 \\
\hline 10 & 20.455 & 27.955 & 1.367 \\
\hline 15 & 31.250 & 40.000 & 1.280 \\
\hline
\end{tabular}

For further details, a relationship between wave generation using linear and nonlinear random wave theory for a long wave record (duration $=4.5 \mathrm{hr}$ ) at all the sea state has been constructed. It was being done by fit the parameter of $\theta$ in Eqs. (10) and (15) at 0 , and $\pi / 2$. Based on the correlation coefficient in Table 2, it is obvious that the relationship between those theory getting poor as the the angle between the wave component more closer. While based on the sea state condition, low sea state experienced better correlation compared to higher sea state. For a better illustration, Figs. 8 and 9 illustrated a relationship with a good and poor correlation, respectively.

Table 2. Relationship between the surface elevation of linear and nonlinear wave theory

\begin{tabular}{cccc}
\hline $\begin{array}{c}\text { Angle between the wave } \\
\text { component, } \boldsymbol{\theta}\end{array}$ & \multicolumn{3}{c}{ Correlation coefficient } \\
\cline { 2 - 4 } $\boldsymbol{0}$ & $H_{S}=5 \mathrm{~m}$ & $H_{s}=10 \mathrm{~m}$ & $H_{S}=15 \mathrm{~m}$ \\
\hline $\boldsymbol{\pi} / \mathbf{2}$ & 0.649 & 0.613 & 0.375 \\
\hline
\end{tabular}




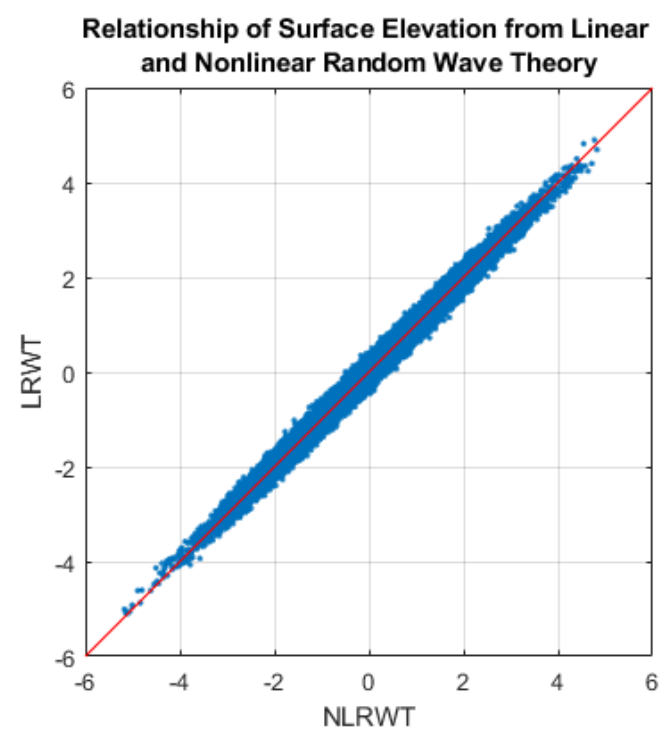

Fig. 8. Relationship of surface elevation using linear and nonlinear wave theory for $H_{s}=5 \mathrm{~m}$, $T_{z}=7.94 \mathrm{sec}, \theta=\pi / 2$ and duration $=4.5 \mathrm{hr}$.

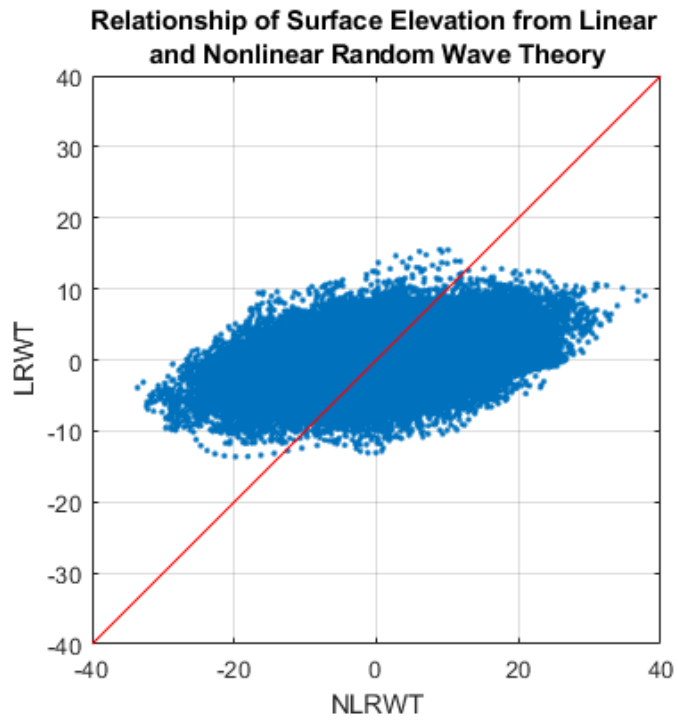

Fig. 9. Relationship of surface elevation using linear and nonlinear wave theory for $H_{s}=15 \mathrm{~m}$, $T_{z}=13.75 \mathrm{sec}, \theta=0$ and duration $=4.5 \mathrm{hr}$.

Hence, based on the sea state condition, the better relationship of surface elevation using both wave theory at low sea state compare to the higher one may result on the nearest to the unity in the ratio of prediction extreme values. However, the result represents the opposite way. Yet, this was related to the random values of $\theta$ as lies in between 0 to $\pi / 2$. The results showing that an angle of interaction between two wave component give a significant effect on the prediction of 100-year wave height. At low sea state condition, the combination of two waves components are prone to experience small changes in the angle of its interaction producing poa or relationship between those theory and cause a huge gap in the prediction of 100-year wave height. In contrast, higher sea state condition allowed 
the interaction between two waves to have a huge angle. As an angle approaching $\pi / 2$ give the good relationship between both theories, its ratio for the prediction of 100 -year wave height was almost unity.

\section{Conclusion}

This paper investigated the behavior of wave generation using linear and nonlinear random wave theory based on the prediction of the 100-year wave height. By considered the nonlinearities based on the contribution of second-order correction term, the higher prediction is obtained in the probability distribution of 100-year wave height. Thus proving that the application of linear wave theory underestimates the prediction of 100 -year wave height. Besides, based on further examined on the relationship of wave generation between both theory showing that low sea state provides better agreement compared to higher sea state and as the angle between the interaction of two waves increase, the relationship also obtained better agreement. Since the 100-year wave height is linear related to load response, it can be concluded that the contribution of second-order correction term leads to high load response. However, in the computation of response, the nonlinearities increase once current is presence as it leads to dominant drag responses. This will be discussed further in the next publication.

This work was supported by the Universiti Teknologi Malaysia [grant no: Q.K130000.2540.09H39 / Q.K130000.2540.09H42] and the Ministry of Higher Education (Malaysia) [grant no: R.K130000.7940.4F584 / R.K130000.7840.4F583].

\section{References}

1. S. K. Chakrabarti, Handbook of Offshore Engineering, First., I. Netherlands, UK: Elsevier, (2005)

2. O. Hagen, Guideline for Offshore Structural Reliability Analysis: Application to Jacket Platforms, DNV Technical Report, (1996)

3. P. W. Cheng, A Reliability Based Design Methodology for Extreme Responses of Offshore Wind Turbines, Delft Univ. Wind Energy Res. Inst., p. 238, (2002)

4. M. K. Abu Husain, Determination of the 100-year Waveheight for the Forties Field in the North Sea, Res. Gate Publ., no. September, (2015)

5. J. R. Morison, J. W. Johnson, S. a. Schaaf, The Force Exerted by Surface Waves on Piles, J. Pet. Technol., 2, 5, pp. 149-154, (1950)

6. X. Y. Zheng, Nonlinear Stochastic Response of Offshore Structures: With Focus on Spectral Analysis, in CESOS Highlight Conference \& AMOS Vision, (2013)

7. S. E. Abdel Raheem, Study on Nonlinear Response of Steel Fixed Offshore Platform Under Environmental Loads, Arab. J. Sci. Eng. 39, 8, pp. 6017-6030, (2014)

8. S. Saeedfar, A. K. Abd Wahab, An Evaluation of the Depth of Similarity between Linear and Non-linear Wave Theories, in 3rd International Conference on Water Resources, pp. 1$11,(2015)$

9. A. Alberello, A. Chabchoub, O. Gramstad, A. Babanin, and A. Toffoli, Statistics of Wave Orbital Velocities in Random Directional Sea States, in 19th Australasian Fluid Mechanics Conference, no. December, pp. 8-11, (2014)

10. G. Z. Forristall, Wave Crest Distributions: Observations and Second-Order Theory, J. Am. Meteorol. Soc., 30, 8, pp. 1931-1943, (2000) 
11. K. Hasselmann, On the Non-linear Energy Transfer in a Gravity Wave Spectrum. Part 1: General Theory, J. Fluid Mech.,12, pp. 481-500, (1962)

12. M. S. Longuet-Higgins and R. W. Ste wart, Radiation Stress and Mass Transport in Gravity Waves, with Application to 'Surf beats', J. Fluid Mech., vol. 13, pp. 481-504, (1962)

13. M. S. Longuet-Higgins, The Effect of Non-linearities on Statistical Distributions in the Theory of Sea Waves, J. Fluid Mech., 17, pp. 459-480, (1963)

14. J. N. Sharma, R. G. Dean, Second-Order Directional Seas and Associated Wave Forces, Soc. Pet. Eng. J., 21, 1, pp. 129-140, (1981)

15. J. F. Dalzell, A note on finite depth second-order wave-wave interactions, Appl. Ocean Res., 21, 3, pp. 105-111, 1999.

16. P. A. E. M. Janssen, M. Onorato, The Shallow Water Limit of the Zakharov Equation and Consequences of (Freak) Wave Prediction, United Kingdom, (2005)

17. J. Chen, Nonlinear Wave Loads on Offshore Wind Support Structure. Master of Science Thesis, Norwegian University of Science and Technology, (2014)

18. T. A. A. Adcock, The Second Order Contribution to Wave Crest Amplitude - Random Simulations and NewWave, in Proceedings of the 25th International Ocean and Polar Engineering Conference, (2015)

19. H. Mallahzadeh, Y. Wang, M. K. Abu Husain, N. I. Mohd Zaki, G. Najafian, 'Efficient Derivation of the Probability Distribution of Extreme Responses due to Random Wave Loading From the Probability. in ASME 32nd International Conference on Ocean, Offshore and Arctic Engineering, (2013)

20. H. Mallahzadeh, Y. Wang, M. K. Abu Husain, N. I. Mohd Zaki, and G. Najafian, Accurate Estimation of the 100-Year Responses From the Probability Distribution of Extreme Surface Elevations, in ASME 33rd International Conference on Ocean, Offshore and Arctic Engineering, (2014)

21. G. B. Airy, Tides and Waves, Reprint. J.J Griffin, (1952)

22. S. R. Massel, Ocean Surface Waves: Their Physics and Prediction, Reprinted. World Scientific Publishing Co. Pte. Ltd, (2005)

23. P. Anderson, Wave Interference, Bozeman Science, (2015)

24. J. N. Sharma and R. G. Dean, Second-Order Directional Seas and Associated Wave Forces, in Offshore Technology Conference, pp. 2505-2510, (1979)

25. A. Toffoli, J. Monbaliu, M. Onorato, A. R. Osborne, A. V. Babanin, E. Bitner-Gregersen, Second-Order Theory and Setup in Surface Gravity Waves: A Comparison with Experimental Data, J. Phys. Oceanogr., vol. 37, no. 11, pp. 2726-2739, (2006)

26. B. Lautrup, Stokes waves, in Physics of Continuous Matter, Second Edi., The Neil Bohr Institute, pp. 1-12, (2011)

27. K. K. Varma, Finite Amplitude Ocean Waves: Waves with Peaked Crests and Broad Troughs, Gen. Artic., no. November, pp. 1047-1057, (2014)

28. D. Majewski, M. Paprota, M. Szmytkiewicz, Water wave measurements at Bellsund in the western Spitsbergen, no. January, (2016) 